\title{
THE COMPLICATIONS AND TREATMENT OF STAPHYLOCOCCAL SEPTICAEM1A IN CHILDHOOD
}

\author{
By Ronald J. Cantwell, M.B., M.R.C.P.(Ed.), D.C.H. \\ Formerly, House Physician, Hammersmith Postgraduate Hospital, London
}

The complications of staphylococcal septicaemia are many and varied, according to the age group affected.

In the adult, acute bacterial endocarditis, pneumonia (which may be further complicated by acute pyogenic pericarditis), osteomyelitis and focal abscess formation in the liver, spleen, kidney and brain may all occur.

In childhood, staphylococcal pneumonia with its attendant danger of pyopneumothorax is the commonest complication in the first 18 months of life. Osteomyelitis may also occur. In infancy, it may occur as a benign condition, or it may be a severe illness endangering life (Thomson and Lewis, 1950). Pericarditis and focal abscess formation are much less common complications in childhood. Multiple complications of staphylococcal septicaemia are unusual.

Impink et al. (1943) described a case of staphylococcal septicaemia with secondary osteomyelitis, acute purulent pericarditis, pneumonia and pleural effusion in a four-year-old girl. Treatment with sulphadiazine, bacteriophage, blood transfusions and pericardial aspiration resulted in recovery. Rohmer et al. (1946) successfully treated an i8month-old child who had staphylococcal septicaemia, osteomyelitis and pericarditis with large doses of iodine and sulphonamides. The only untoward effects were transient haematuria and occasional vomiting.

Zimmerman et al. (1946), Bret et al. (1946) and Coffey (1948) described cases of pericarditis complicating staphylococcal septicaemia in adults in which recovery followed treatment with penicillin. In Coffey's case staphylococcal pneumonia preceded the onset of pericarditis.

Terrasse et al. (I945) successfully treated a boy of Io years who developed osteomyelitis of the tibia, pneumonia and pericarditis complicating staphylococcal septicaemia. Penicillin and sulphonamides, together with pericardial aspiration, were necessary.
Jones-Davies et al. (1950) also described a case of suppurative pericarditis complicating osteomyelitis of the femur on the tenth day. The case, a boy of ro years, was successfully treated with streptomycin systemically and intrapericardially.

The present case illustrates some of the complications that may occur in a child.

\section{Case Report}

L.P., aged three years, presented with a history of fever, drowsiness and general malaise of $3^{6}$ hours' duration. In the 12 hours prior to admission there had been anorexia, vomiting, sweating and pain in the left leg.

The past history was non-contributory.

On examination: Temperature $103.6^{\circ} \mathrm{F}$., pulse I40 per minute. A flushed, fretful, drowsy boy exhibiting a toxic delusionary state. There were numerous infected scratches and abrasions on the knees, elbows and hands. Kernig's sign negative. Reflexes brisk. Some limitation of external rotation of left hip, but no localizing tenderness elicited. B.P. 100/0. Soft systolic murmur over praecordium. Femoral pulses palpable. Liver and spleen not enlarged.

A diagnosis of septicaemia (probably staphylococcal) was made.

\section{Investigation, Progress and Treatment}

On admission a lumbar puncture was performed because of the drowsiness and vomiting, but this produced a clear colourless fluid under normal pressure. Blood was also taken for culture and whilst awaiting a report symptomatic treatment with 'Disprin' was commenced.

By the following morning the temperature had risen to $105.6^{\circ} \mathrm{F}$. and the left hip was being held in flexion and external rotation. Heavy percussion of the left heel was not painful, however. A diagnosis of osteomyelitis of the neck of the left femur was made and after a further blood culture, crystalline penicillin was commenced in doses of 500,000 
units eight-hourly. To lessen muscle spasm and relieve pain the left leg was put in a Thomas splint with skin traction.

At this time, Hb. Io g. (68 per cent.), W.B.C. $\mathrm{I} 8,000 / \mathrm{cu}$. $\mathrm{mm}$. with 84 per cent. neutrophils, I3 per cent. lymphocytes, 3 per cent. monocytes. A throat swab grew normal flora and the urine contained no pus cells or organisms.

By the second day after admission the blood culture had grown Staphylococcus aureus. The child was still very ill, but now showed a definite fullness in the left groin, which was very tender to touch, and movements of the left hip were much restricted. X-ray of the chest and hip joints showed no abnormality.

On the following day the staphylococcus isolated from the blood was shown to be resistant to penicillin, but sensitive to streptomycin, tetracycline, chloramphenicol and erythromycin. As a result of this, penicillin was replaced by chlortetracycline, $500 \mathrm{mg}$. daily in divided doses. The tuberculin jelly test at this time was negative.

On the sixth day the second blood culture had also grown Staphylococcus aureus with identical sensitivities. W.B.C. I3,000/cu. mm. with neutrophil predominance.

The temperature and pulse fell gradually over the next few days with some symptomatic improvement. On the eighth day what appeared to be an infected hydrocele developed on the right side. The overlying skin was oedematous and very tender to touch and as the child was still very ill streptomycin was added in a dose of $250 \mathrm{mg}$. b.d. On the tenth day $\mathrm{Hb}$. 68 per cent., W.B.C. 10,000/cu. mm. with 63 per cent. neutrophils, 27 per cent. lymphocytes.

By the fourteenth day the swelling of the left groin and the scrotal swelling had subsided. However, a rough pericardial friction rub was now audible over the whole praecordium and the cardiac dullness extended into the axilla. A diagnosis of pericarditis with effusion was confirmed by chest $\mathrm{X}$-ray. The latter also revealed a small patch of consolidation in the right mid zone. There was no evidence of cardiac tamponade at the time. The spleen was now palpable 2 F.B. below the costal margin. A further blood culture at this time was sterile.

On the following day, although there was no dyspnoea, the jugular venous pressure was elevated moderately and a presystolic triple rhythm was audible at the apex. Further X-rays of the hips now showed definite osteoporosis of the upper end of the left femur and acetabulum.

On the sixteenth day, because of increasing dyspnoea, $160 \mathrm{ml}$. of slightly blood-stained opalescent fluid was aspirated from the pericardial cavity and $50 \mathrm{mg}$. erythromycin in $10 \mathrm{ml}$. of 0.9 per cent. saline was instilled. At the same time, systemic erythromycin was commenced, $500 \mathrm{mg}$. daily in divided doses. Streptomycin was con- $\triangle$ tinued with the erythromycin, but chlortetra-.. cycline therapy was terminated.

Culture of the pericardial fluid grew Staphylo $-\frac{\vec{P}}{\frac{D}{O}}$ coccus aureus (scanty growth). Identical sensitivities to the staphylococci isolated from the blood were $\frac{\bar{c}}{\mathrm{~s}}$ obtained. Chest X-ray following pericardial $\vec{\Phi}$ aspiration showed a smaller cardiac silhouette with clear lung fields.

By the following day the dyspnoea had markedly $\overrightarrow{0}$ improved, although the fever persisted. An E.S.R. at this time was $120 \mathrm{~mm}$. in one hour, and the $\vec{\omega}$ W.B.C. was $14,000 / \mathrm{cu}$. mm., but a further bloodo culture was also sterile. On the twentieth day the urine contained leucocytes +-+ and erythro- $-\dot{\omega}$ cytes ++ but culture of same was sterile. The + $\mathrm{X}$-ray of the pelvis now showed an area of $\omega$ osteoporosis in the ascending ramus of the left pubic bone with some surrounding periosteal reaction.

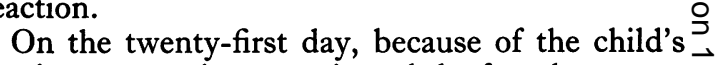
persistent pyrexia, anaemia and the fact that meta- $c$ static foci were occurring despite adequate doses $\mathbb{D}$ of the appropriate antibiotics, a transfusion of $\overrightarrow{\mathbb{D}}$ $250 \mathrm{ml}$. of fresh blood was given. This resulted in a rise in the haemoglobin from 68 to $88 \mathrm{pg} \stackrel{\mathbb{D}}{-}$ cent., a striking improvement in the patient's wei $\overrightarrow{0}$ being and a fall in temperature. By the twent 0 fourth day the urine contained only a few leuco cytes, the culture being sterile. However, the benefits of the fresh blood were only short-lived, as on the twenty-eighth day the temperature had risen to $100^{\circ} \mathrm{F}$. in the evening, and a further W.B.C. was $\frac{\circ}{\mathbb{D}}$ $\mathrm{I} 8,000 / \mathrm{cu}$. mm. Because of this, both streptomycin and erythromycin were discontinued and $\bar{O}$ tetracycline was commenced in a dose of $500 \mathrm{mg}$. daily. This resulted in a fall in the white count on the thirty-fifth day to $14,000 / \mathrm{cu}$. mm., of which 60 per cent. were now lymphocytes.

Further X-rays on the thirty-eighth day showed an almost normal cardiac silhouette and the focus in the left pubic bone to be unchanged.

On the forty-eighth day the heart size was now normal, but the focus of osteoporosis was now 0 smaller. By this time the child was afebrile and very active. As no weight-bearing bone was? involved, ambulation was commenced. Four days later the white count was $10,000 / \mathrm{cu} . \mathrm{mm}$., of $\sigma$ which 50 per cent. were lymphocytes, and on the N fifty-fourth day tetracycline therapy was discon- N tinued. Three days later, the fifty-seventh day $\sigma$ after admission, the child was discharged to $a_{0}$ convalescent home for four weeks.

\section{Discussion}

In this case it seems more than probable that 
septic abrasions of the skin provided the portal of entry for the Staphylococcus aureus. The resultant septicaemia was soon complicated by pubic osteitis. Haematogenous osteitis involving the pelvic bones, as in this instance, is an unusual complication, long bone involvement being much the more common. Trueta and Agerholm (1946) cited three instances of pelvic involvement amongst 37 cases of osteomyelitis, an incidence of 8.I per cent. White and Dennison (1952) had eight cases with pelvic osteitis in a series of 212 cases of acute haematogeneous osteitis ( 3.7 per cent.). Green et al. (1956) had similar figures, pelvic involvement occurring in four instances among 99 patients with osteomyelitis (4 per cent.).

The occurrence of acute staphylococcal pericarditis preceded by pneumona (almost certainly staphylococcal) further complicated this case, although blood cultures at the time were sterile.

Presumably at this time there was a transient shower of staphylococci into the blood stream from the pyogenic focus in the pelvis, and by the time this metastatic focus had become established in the right lung the blood was now sterile. Spread by direct continuity to involve the pericardium thence resulted in the appearance of acute pyogenic staphylococcal pericarditis. Osteomyelitis followed later by pneumonia and pericarditis occurred in the cases described by Impink et al. (1943) and Terrasse et al. (1945) and further confirms the well-known fact that acute staphylococcal pericarditis usually occurs secondary to staphylococcal pneumonia.

The occurrence of profuse pyuria on the twentieth day with a sterile urine culture on the following days suggests that a metastatic focus had formed in one kidney. This had been rendered sterile by antibiotic therapy and thus its ultimate rupture into the pelvis of the ureter resulted in a sterile pyuria and spontaneous cure.

The striking, if only transient, improvement in the patient's general condition following the fresh blood transfusion suggests that frequent small transfusions would be well worth while in the seriously ill patient who is only just holding his own despite the appropriate antibiotics.

\section{Conclusions}

Despite all the modern antibiotics at our disposal, staphylococcal septicaemia remains a serious illness and, together with its complications, constitutes a grave hazard to life.

It is well to consider the possibility of pyogenic metastases to bone in any patient who presents with staphylococcal septicaemia. It is also advisable to examine very ill patients, especially infants, periodically for evidence of pyogenic bone involvement. Localized bone tenderness due to elevation of the periosteum and hyperaesthesia overlying the lesion are useful localizing signs. The discovery of such involvement should result in prolonged and intensive treatment with the appropriate antibiotic until the bony infection has been completely eradicated.

Repeated examinations of the chest and urine will detect early pulmonary cardiac or renal complications.

The use of fresh blood transfusions is thoroughly justified in the more severe cases.

\section{Summary}

A case of staphylococcal septicaemia in a child, with its complications and treatment, is described. Complications included pelvic osteitis, staphylococcal pneumonia, acute pyogenic pericarditis and renal abscess formation. Similar cases occurring in the literature over the past 16 years are reviewed.

\section{BIBLIOGRAPHY}

BRET, J., and VINCENT, E. (1946), Arch. Mal. Coeur, 39, 29 r. COFFEY, J. (1948), Irish f. med. Sci., No. 270, 274.

GREEN, M., NYHAN, W. L. (Jnr.), and FOUSEK, M. D. (1956), Pediatrics, 17, No. 3, 368

IMPINK, R., DENHOFF, E., and VANDER VEER, J. B. (1943), Amer. Heart F., 26, 699.

JONES-DAVIES, T. E., and CAMPBELL, C. (1950), Brit. med. $\mathscr{~ . , ~}$ i, 1350.

ROHMER, P., SACREZ, R., and BEYER, P. (1946), Arch. franf. Pédiat., 3, 73 .

TERRASSE, J., LERE, J., and BOUCHARENC, G. (r945), Bull. Soc. méd. Hóp. Paris, 6r, 400 .

THOMSON, J., and LEWIS, I. C. (1950), Arch. Dis. Childh., 273.

TRUETA, J., and AGERHOLM, M. (1946), Lancet i, 877.

WHITE, M., and DENNISON, W.M. (1952), f. Bone, f. Surg. 34B, 608 .

ZIMMERMAN, J., DURGIN, B., (1946), Amer. Heart f., 3r, 93. 